\title{
Accuracy Simulation Method Based on Assembly Process Information Mapping
}

\author{
Jianjun Tang ${ }^{1}$ and Haitao Liu ${ }^{1}$ \\ ${ }^{1}$ AVIC Chengdu Aircraft Industry (Group) Co., Ltd., Chengdu, 610092, P.R. China
}

\begin{abstract}
Assembly process information has a direct impact on product manufacturing accuracy. In order to accurately predict product accuracy, a simulation method based on assembly process information mapping was proposed. The assembly deviation information was expressed as the in-part deviation reference, feature deviation Vector direction, feature deviation vector size, deviation transmission connection form between the parts and the deviation transmission direction, and so on. The mapping between the assembly process information and the deviation transmission information was established. Then the assembly accuracy simulation algorithm including the main algorithm and assembly deviation transfer model building algorithm was established. Taking C919 internal flap assembly as an example, the effectiveness of this method had been verified.
\end{abstract}

\section{Introduction}

Aircraft assembly accuracy is an important factor affecting the performance of the whole machine. Due to the different assembly constraints between the parts, the dimensions deviation of the parts has a different degree of influence on the overall assembly accuracy [1]. Because the assembly process defines the assembly sequence of parts and assembly constraints, so In order to obtain more reliable simulation results of assembly accuracy, the information of assembly process needs to be fully integrated.

The assembly process defines all the information that can reflect the deviation transfer path and directly affect the formation of assembly accuracy [2-4]. Literature [5-7] studied the assembly process information model and the tolerance information model, but lacks the research on the relationship between the two.

In order to analyze the influence of assembly process information on the assembly accuracy comprehensively and obtain a more reliable prediction value of assembly accuracy, a simulation method based on assembly process information mapping is proposed. Through the construction of the mapping between multi-factor assembly process information and assembly deviation transfer information, as well as the assembly accuracy simulation algorithm to achieve a reliable assembly accuracy simulation.

\section{Multi-factor assembly information definition}

Improper tolerance allocation or unreasonable process planning will lead to over-accuracy of assembly key characteristics. The existing aircraft tolerance distribution is mainly based on experience and knowledge. There is no systematic analysis of the factors influencing the accuracy of the assembly key features in the assembly process. It is difficult to find out the accuracy of the assembly key characteristics before the aircraft enters the assembly production.

According to the characteristics of aircraft assembly process planning, assembly process consists of multiple assembly processes. By extending the assembly process model, the assembly process information related to assembly deviation transmission is analyzed, which mainly includes product model information, process design information and tooling model information. Product model information describes product design dimensions and tolerance; process design information describes assembly sequences, assembly features, assembly constraints, and positioning references; tooling model information describes tooling dimensions and tolerances.

\section{Mapping relationship building}

In order to establish a more realistic transfer path of assembly deviation, it is necessary to determine the mapping relationship between multi-factor process information and deviation transfer information.

\subsection{Abstract Expression of Assembly Deviation Transfer Information}


Assembly deviation transmission refers to the assembly deviation along the direction of deviation transmission to reach the key characteristics of the assembly process nodes. Assembly deviations are propagated once at each deviation transfer node. Deviations are mainly composed of two parts: the own deviation of the node and the variation deviation of the previous node. Deviation can be expressed as

$$
T_{i+1}=a_{i} \cdot T_{i}+t_{i+1}
$$

Where:

$T_{i}$ said the previous node's deviation value;

$a_{i}$ represents the coefficient of variation transmitted by $T_{i}$ to the next node, which is determined by the deviation transfer path;

$t_{i+1}$ indicates the own deviation value of this node;

$T_{i+1}$ indicates the deviation value formed by this node.

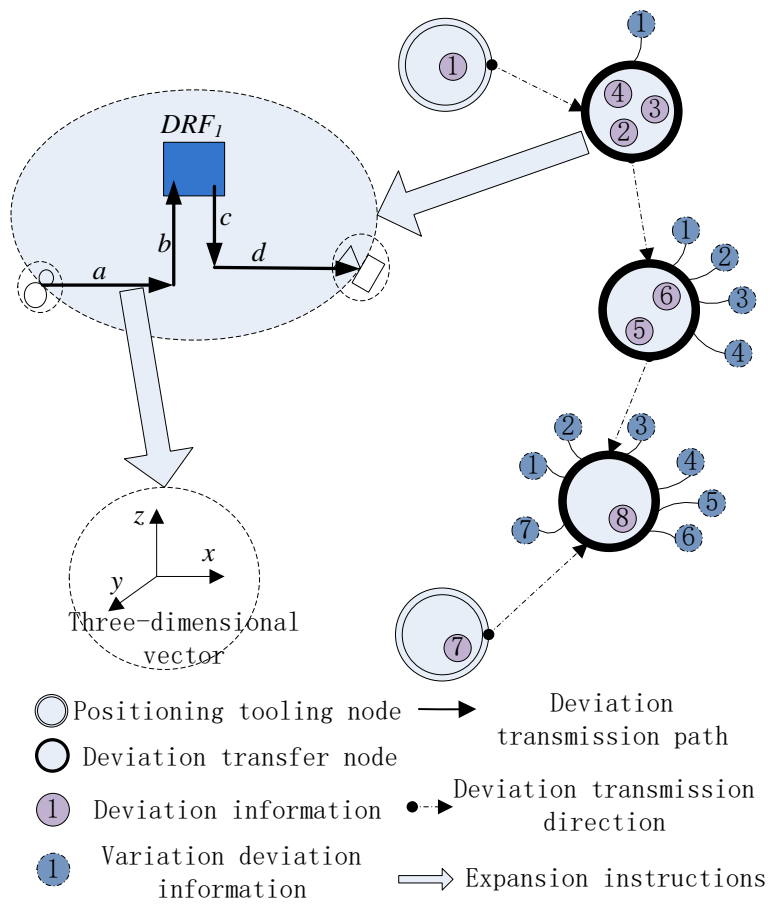

Fig. 1 Assembly deviation transfer information

Assembly deviation transfer information abstract expression as shown in Fig. 1. Aircraft assembly process is generally located by the tooling, so the initial node of assembly deviation transmission is positioning tooling node. The assembly deviation transfer process mainly passes two types of deviations: the deviations introduced by this node and the deviations introduced before this node. Within each deviation transfer node, the deviation is transmitted in a certain deviation transfer path, which must go through the in-part deviation reference, which is generally the part positioning reference. The path of deviation transmission is determined by the design dimensional information of the part, which mainly contains the information of three-dimensional vector size and direction.

Assembly deviation transfer information is affected by product design information and process information. And they have corresponding relationships. By discussing the mapping relationship between the assembly process information and the deviation transfer information, a more reliable deviation transfer path can be obtained, which is the basis of the assembly accuracy simulation.

\subsection{The Mapping Relationship for Assembly Deviation Transfer}

According to the analysis of pheromone information of assembly deviation transmission, the assembly deviation transmission model mainly includes five types of information: in-component deviation reference standard, characteristic deviation vector direction, characteristic deviation vector size, deviation transmission connection form between parts and deviation transmission direction. These five types of information have one-to-one correspondence with the positioning datum, assembly characteristic, design tolerance, assembly constraint and assembly sequence in MBOM, as shown in Fig. 2.

\section{Assembly accuracy simulation based on Monte Carlo}

Through the assembly accuracy simulation can fully analyze the assembly process on the accuracy of key features. Before actual assembly and assembly, the input condition of assembly accuracy simulation is the design tolerance zone. How to divide the design tolerance zone into a set of effective deviation values is a prerequisite for assembly simulation. The use of Monte Carlo algorithm can effectively solve the above problems.

The simulation of assembly accuracy based on Monte Carlo algorithm is shown in Fig. 3. The main work includes building the assembly deviation transfer model and setting the correlation value of the Monte Carlo algorithm. The main steps of the algorithm are as follows:

Step 1: Determine the key features that need to be simulated, using this as a basis to resolve the associated assembly units, thereby reducing the amount of computation;

Step 2: Determine if the corresponding assembly deviation transfer model has been constructed

If Yes Jumping to step 3 to continue execution;

If No Jumping to assembly deviation transfer model building algorithm;

Step 3: Define the number of deviation samples $n$, setting $\mathrm{n}=5000$;

Step 4: Enter the tolerance field for all relevant features, and use the random number generation principle to generate a deviation random sample matrix of key features;

Step 5: Calculate the distribution of the simulation results and determine whether the deviation within \pm 3

$\sigma$ is out of tolerance;

If No Then come to meet the accuracy requirements of the results;

If Yes The assembly process need to be optimized.

Assembly deviation transfer model building algorithm specific steps are as follows: 
Step 1: Analyze the assembly constraints, the associated assembly features and positioning features in the corresponding assembly unit;

Step 2: Build the deviation reference base on the location feature;

Step 3: The characteristics and the direction of the deviation transmission are expressed by vectors;

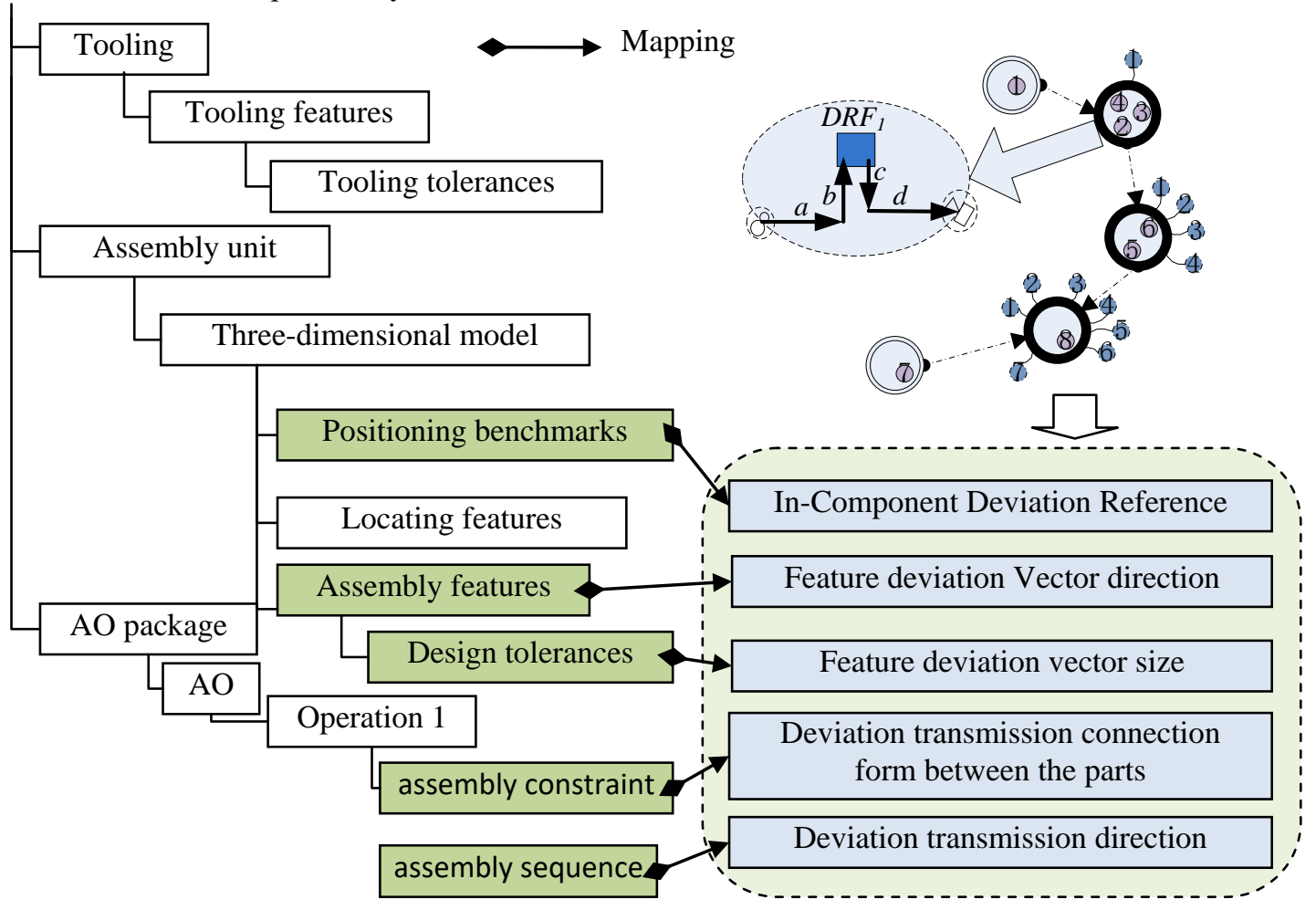

Assembly process model
Step 4: In the corresponding assembly unit, according to the assembly constraints to build the relationship between the parts of the deviation transfer connection.

Step 5: Return to the main algorithm.

Fig. 2 The mapping between assembly process information and deviation transmission information

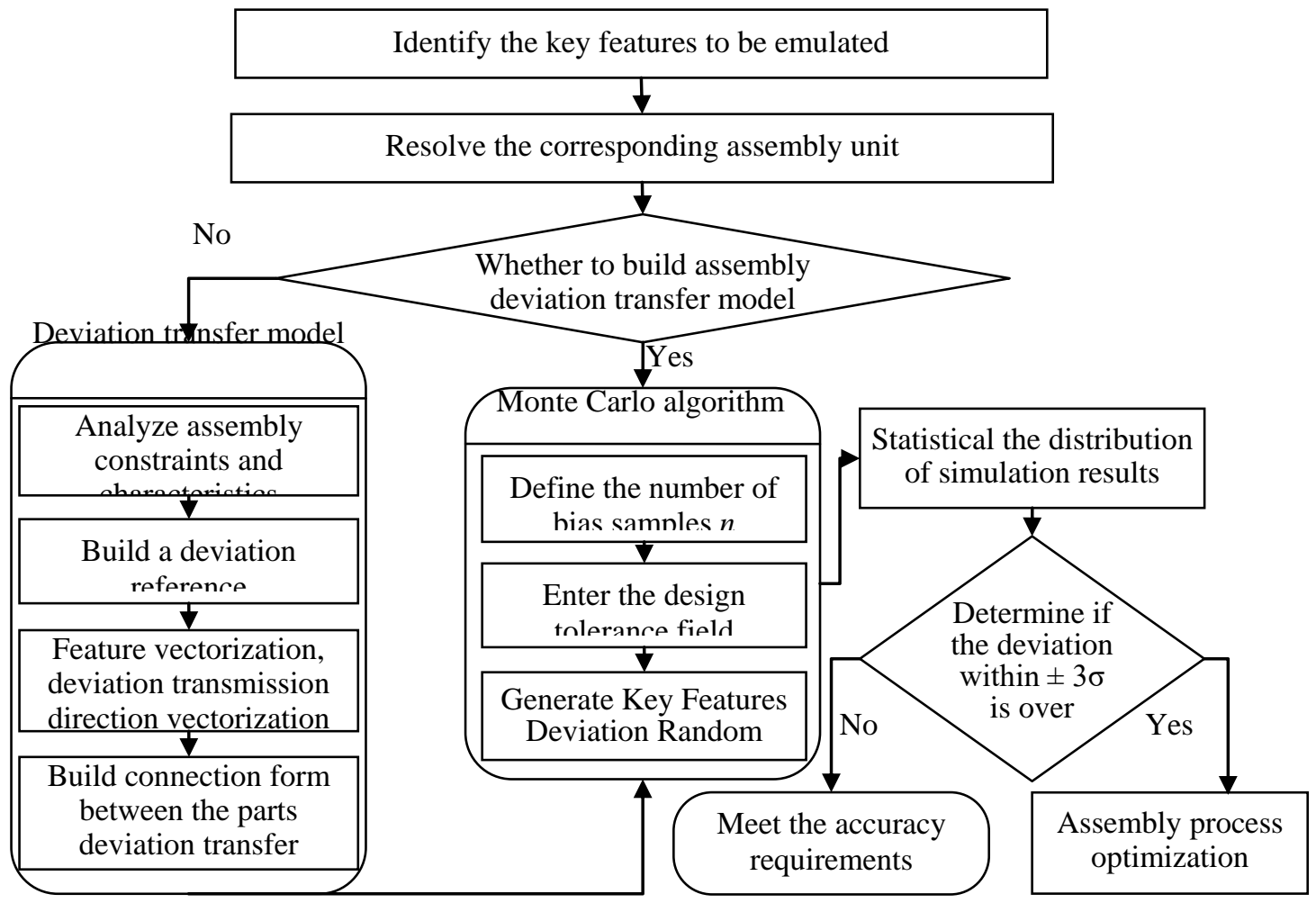

Fig. 3 Assembly Accuracy Simulation Based on Monte Carlo Algorithm 


\section{Examples of verification}

Taking the C919 inner flap component assembly as an example, an assembly accuracy simulation process based on assembly process information mapping is described. The assembly constraints contained in the assembly unit of the inner flap components are analyzed; the corresponding assembly features and positioning features are constructed; According to the vector direction and size of the corresponding surface in the 3D model of the product, a corresponding assembly deviation transfer model is constructed, shown in Fig. 4.

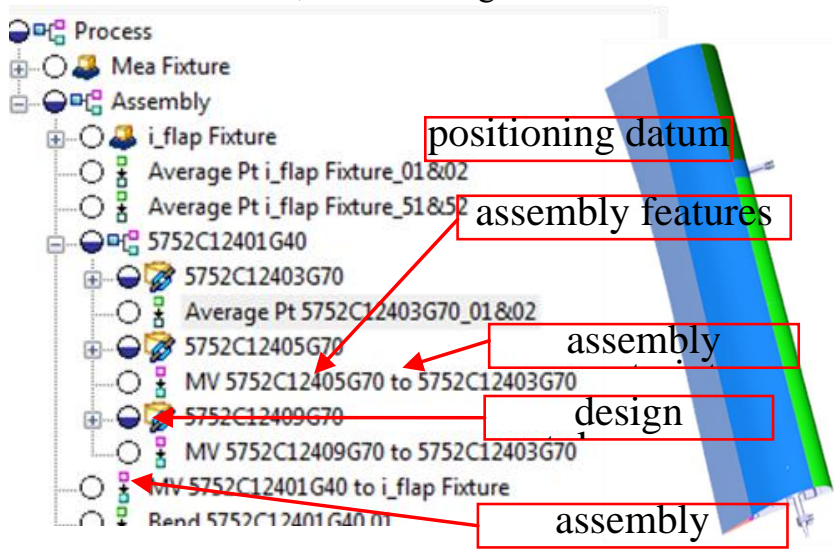

Fig. 4 Inner flap assembly deviation transfer model

The accuracy simulation results of the target key features included in the inner flap components are shown in Fig. 5.

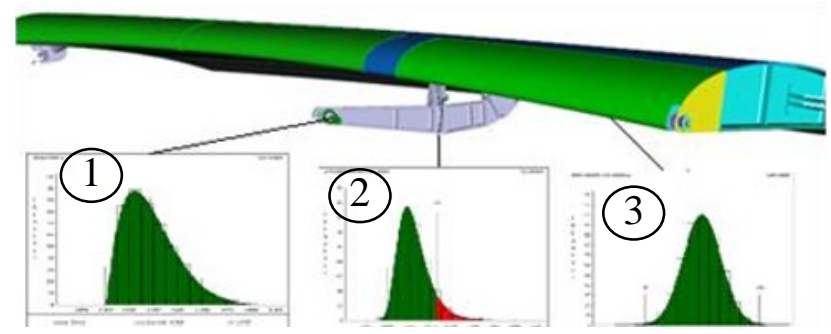

Fig. 5 Slab components target key features of precision simulation results

In Fig. 5, (1), (2) and (3) represent the statistic results of the simulation results of the inner profile flaps. It can be seen from the simulation results in the figure that there is a possibility that the axis deviation of the 2 \# pulley carrier tab joint hole may be out of tolerance. By optimizing the assembly process, assembly precision can be effectively controlled.

\section{Summary}

Through establishing the mapping relationship between assembly process information and assembly deviation transfer information, a real assembly deviation transfer model is constructed. The method combines the effects of positioning datum, assembly features, design tolerances, assembly constraints, and assembly sequences on assembly deviation transfer. Using the assembly precision simulation based on Monte Carlo algorithm, the accurate prediction of the assembly key characteristics can be realized.

\section{References}

1. Jin, J. (2005). Process-oriented tolerancing for multistation assembly systems. Iie Transactions, 37(6), 493-508.

2. Xu, S., \& Keyser, J. (2014). Geometric computation and optimization on tolerance dimensioning. Butterworth-Heinemann.

3. Samuel H. Huang, Qiu Liu, \& Rami Musa. (2004). Tolerance-based process plan evaluation using monte carlo simulation. International Journal of Production Research, 42(23), 4871-4891.

4. Hong, Y. S., \& Chang, T. C. (2002). A comprehensive review of tolerancing research. International Journal of Production Research, 40(11), 2425-2459.

5. Bing, Y., \& Zhang, L. X. (2006). Introduction of integrated assembly model for assembly process planning. Journal of System Simulation.

6. Liu, Y. (2003). Hierachical representation model and its realization of tolerance based on feature. Chinese Journal of Mechanical Engineering, 39(3), 649-50.

7. Guilford, J., \& Turner, J. (1993). Representational primitives for geometric tolerancing. ComputerAided Design, 25(9), 577-586. 